\title{
Análisis bibliométrico de las tesis doctorales sobre Internet en las universidades españolas (1996-2011)
}

\author{
A bibliometric analysis of doctoral dissertations \\ about the Internet in Spanish \\ universities (1996-2011)
}

Jesús DÍAZ-CAMPO'

\section{Resumen}

El presente trabajo es un análisis descriptivo de las tesis doctorales sobre Internet que se han defendido en las universidades de España entre 1996 y 2011. La fuente principal es la base de datos Teseo del Ministerio de Educación, cuya información se ha completado con otras fuentes. El número total de tesis defendidas en el periodo estudiado es de 1020 títulos. La metodología empleada es el análisis bibliométrico. Se analiza la producción según el sexo del autor, el año de lectura, el idioma, el área de conocimiento, la universidad y departamento de lectura, los directores y los miembros de los tribunales evaluadores, y los datos se comparan con los obtenidos por Fuente, y Arguimbau, en su estudio sobre la producción total de tesis doctorales en España. Los resultados muestran que la producción total se reparte entre 62 universidades; el área de conocimiento en el que se han defendido un mayor número de tesis es el de las Ciencias Sociales y Jurídicas. Por último, destaca el número de tesis que han sido codirigidas.

Palabras clave: Bibliometría. España. Internet. Producción científica. Tesis doctorales.

\begin{abstract}
This is a descriptive study about doctoral dissertations on the Internet which have been defended in Spanish universities between 1996 and 2011. The main source of information has been the Teseo database of the Ministry of Education, whose information has been completed with other sources. The total number of dissertations produced during the studied period was 1,020. Bibliometric analysis techniques have been used in order to analyze the data. Production is analyzed for PhD candidate genre, time period, theses language, topic, universities and departments, theses advisors and examination board members. These data are compared with those obtained by Fuentes and Arguimbau when studying the total production of doctoral dissertations in Spain. Results show that the doctoral dissertations have been produced in 62 different universities. Social Sciences and Law is the knowledge area with the highest number of thesis. Finally, there are an important number of doctoral dissertations which have been co-directed.
\end{abstract}

Keywords: Bibliometric studies. Spain. Internet. Scientific production. Doctoral theses.

\section{Introducción}

El surgimiento de Internet ha supuesto uno de los acontecimientos más determinantes en nuestra historia reciente y ha provocado una serie de transformaciones que afectan a todos los ámbitos de nuestra vida, empezando por la forma en la que nos relacionamos con los demás. A partir de su consolidación en la década de

\footnotetext{
1 Universidad Internacional de La Rioja, Facultad de Ciencias Jurídicas, Sociales y Humanidades, Grupo de Investigación Comunicación y Sociedad Digital. Av. de la Paz, 137, 26006, Logroño, La Rioja, España.E-mail: <jesus.diaz@unir.net>.

Recebido el 7/7/2015, re-presentado el 11/11/2015 y aceptado para su publicación el 17/12/2015.
} 
los 1990 del siglo XX, las investigaciones en el mundo académico referidas a Internet han sido muy numerosas y se han llevado a cabo con enfoques muy diferentes. El presente trabajo pretende acercarse a esa misma cuestión desde una perspectiva distinta.

Las tesis doctorales constituyen una de las vías para analizar las diversas líneas de investigación que se están desarrollando en un campo concreto de conocimiento, así como sus características principales, la evolución que están siguiendo y el grado de consolidación alcanzado. Asimismo, la importancia del estudio y análisis de la tesis doctorales radica en que "permite a la comunidad científica situarse frente a una información que aún sabiendo que existe no se valora en su conjunto"(Sánchez-Vigil et al., 2014, p.10).

En España se han publicado numerosos análisis bibliométricos sobre tesis doctorales. Las referencias de la mayoría de ellos se pueden consultar en el trabajo de Repiso (2013), que recoge hasta 72 análisis de este tipo, y a los que habría que sumar la reciente investigación de Sánchez-Vigil et al. (2014) sobre fotografía o la de Oliva Marañón (2014) sobre Historia del Periodismo. No obstante, cabe mencionar algunas investigaciones que han servido de referencia en este campo, como los de Moralejo Álvarez (2000); Delgado et al. (2006); Fuentes y Arguimbau (2010); o los trabajos del Observatorio de la Actividad Investigadora de las Universidades Españolas (lune), creado precisamente para realizar un seguimiento de la actividad investigadora por parte de las universidades españolas.

Como han puesto de relieve varios de estos estudios (Moralejo Álvarez, 2000; Sorli \& Merlo, 2002; Orera Orera, 2003; Fuentes \& Arguimbau, 2010), ha sido precisamente la eclosión del objeto de estudio de esta investigación - Internet - la que ha facilitado el acceso y la difusión a las tesis doctorales, que hasta hace un tiempo formaban parte de la denominada literatura gris. De hecho, una de las fuentes principales para llevar a cabo todas estas investigaciones ha sido la base de datos bibliográfica Teseo, dependiente del Ministerio de Educación, Cultura y Deporte del Gobierno de España, accesible de forma gratuita a través de Internet <www.mcu.es/teseo> y que proporciona información sobre las tesis doctorales leídas en el país desde 1976.
Sin embargo, son varias las investigaciones (Martínez Pestaña, 2004; 2011a; 2011b; Fuentes \& Arguimbau, 2010; Marcos Recio et al., 2012; Repiso et al., 2012a; 2012b; 2013) que advierten de la falta de exhaustividad de Teseo. En concreto, el rigor con el que los doctorandos y los miembros de los tribunales rellenen los formularios es uno de los principales elementos que determinan esa exhaustividad, de modo que ni siquiera se puede tener garantía de que todas las tesis que se han defendido han sido registradas posteriormente en Teseo. Además, el campo "Descriptores" carece de valor científico debido a la falta de normalización de materias en Teseo (Repiso et al., 2012b). Con todo, esta base de datos es el recurso más completo para llevar a cabo cualquier tipo de investigación sobre tesis doctorales en España (Sorli \& Merlo, 2002).

Para subsanar esas posibles carencias, Fuentes y Arguimbau (2010) compararon previamente los datos sobre tesis doctorales leídas en España que aparecen en el Instituto Nacional de Estadística (INE) y los que recoge Teseo sobre ese mismo parámetro. En aquella ocasión, los autores comprobaron en su estudio que el porcentaje de tesis doctorales leídas en España y recogidas porTeseo se situaba en el 87,3\% y consideraron ese porcentaje suficiente para realizar su investigación.

Por otro lado, a pesar de la existencia de numerosas investigaciones bibliométricas en España, hasta la fecha tan solo se ha llevado a cabo una investigación sobre tesis doctorales sobre Internet en la universidad española, y ésta se circunscribe al campo de la Comunicación (Díaz-Campo, 2014).

Por ello, el objetivo principal de esta investigación es ampliar ese trabajo y darle una perspectiva global abarcando todas las áreas de estudio, y recopilando y analizando al mismo tiempo los datos estadísticos fundamentales relativos a la producción de tesis doctorales sobre Internet defendidas en las universidades españolas. La herramienta metodológica empleada es el análisis bibliométrico; es decir, la aplicación de técnicas cuantitativas para estudiar las características bibliográficas de dichas tesis doctorales.

\section{Procedimientos metodológicos}

La fuente de información principal para la realización de esta investigación ha sido la base de datos 
bibliográfica Teseo, que administra el Ministerio de Educación, Cultura y Deporte, y de la que ya se ha hablado en el apartado anterior. Teniendo en cuenta que, como también se ha explicado, Teseo no es una fuente exhaustiva, siguiendo la metodología ya mencionada de Fuentes y Arguimbau (2010) se han comparado previamente los datos sobre tesis doctorales leídas en España que aparecen en el INE y los que recoge Teseo sobre ese mismo parámetro. Como puede verse en la Tabla 1, el porcentaje de similitud se sitúa en el 92,8\%, un valor que se considera suficiente para llevar a cabo el análisis.

Este dato indica que se ha producido una agilización en el proceso de registro de esas tesis doctorales y aporta una fiabilidad mucho mayor. En otras investigaciones previas, como la llevada a cabo por Repiso et al. (2011), solo se analizaron las tesis defendidas hasta 2007 a pesar de que los datos se recogieron en 2010, ya que el porcentaje de cobertura no alcanzaba el $70 \%$ en alguno de esos años. Pues bien, en este caso, la cobertura es significativamente más alta.

La selección de las tesis analizadas constó de varias fases. La base de datos Teseo contiene los siguientes campos para cada registro: Autor, Título de la tesis, Universidad y Departamento de Lectura, Fecha de lectura, Director o directores de la tesis, Miembros del
Tribunal y Descriptores. Como ya se ha comentado, este último campo carece de valor al no existir una normalización de materias en esta base de datos. Por ello, la estrategia se orientó temáticamente hacia el título y el resumen. En una primera búsqueda exhaustiva se seleccionaron todas aquellas investigaciones que contienen lo vocablos Internet, digital, digitales, online o Web en alguno de esos dos campos o en ambos. La búsqueda se llevó a cabo por periodos de años, ya que el Teseo permite recuperar un máximo de 250 registros por cada consulta y el número total de tesis doctorales que contienen esos términos en esos campos supera ampliamente esa cifra.

A continuación se analizaron en profundidad las tesis seleccionadas y se descartaron todas aquellas en las que se comprobó que Internet no era realmente el objeto de estudio. También se descartaron aquellas en las que el hecho de que ese u otros términos relacionados formaran parte del resumen estaba motivado porque en la investigación se habían empleado fuentes obtenidas de Internet, o bien porque parte de la metodología se había desarrollado online (por ejemplo, con la realización de encuestas a través de una página Web).

Asimismo, se decidió descartar también todas las tesis defendidas durante el curso 2011-2012, ya que en el momento de realizar esta investigación todavía no

Tabla 1. Comparativa de las tesis registradas por Instituto Nacional de Esdadística (INE) y Teseo (España, 1996-2011).

\begin{tabular}{|c|c|c|c|c|}
\hline Curso & Tesis INE & Tesis Teseo & Diferencia & $\%$ \\
\hline 1996-1997 & 6140 & 5969 & 171 & 97,2 \\
\hline 1997-1998 & 5931 & 5850 & 81 & 98,6 \\
\hline 1998-1999 & 5984 & 5193 & 791 & 86,8 \\
\hline 1999-2000 & 6408 & 6109 & 299 & 95,3 \\
\hline $2000-2001$ & 6380 & 5913 & 467 & 92,7 \\
\hline 2001-2002 & 6936 & 6418 & 518 & 92,5 \\
\hline $2002-2003$ & 7467 & 7003 & 464 & 93,8 \\
\hline 2003-2004 & 8176 & 7259 & 917 & 88,8 \\
\hline 2004-2005 & 6902 & 6349 & 553 & 91,9 \\
\hline $2005-2006$ & 7159 & 6315 & 844 & 88,2 \\
\hline 2006-2007 & 7150 & 6380 & 770 & 89,2 \\
\hline $2007-2008$ & 7302 & 6646 & 656 & 91,0 \\
\hline 2008-2009 & 7915 & 7538 & 377 & 95,2 \\
\hline $2009-2010$ & 8596 & 8227 & 369 & 95,7 \\
\hline 2010-2011 & 8915 & 8443 & 472 & 94,7 \\
\hline Total & 107361 & 99612 & 7578 & 92,8 \\
\hline
\end{tabular}

Fuente: Elaboración propia (2015). 
estaban disponibles los datos correspondientes al INE, lo que imposibilitaba contrastar el porcentaje de cobertura de Teseo en ese periodo. De este modo, el número total de tesis analizadas asciende a 1 020, la primera de los cuales se defendió en el año 1996.

A continuación se revisaron los registros seleccionados para llevar a cabo el análisis, ya que contenían diversas omisiones e imprecisiones. En el primer caso, en varios de los registros no aparecían todos los datos relativos a la composición del tribunal (faltaban uno o varios componentes), y lo mismo ocurría con el departamento al cual estaba adscrito el doctorando. Por lo que respecta a las inexactitudes, la fecha que figura por defecto en muchos de los registros de Teseo es el 1 de enero, un día no lectivo en España y en el que, por tanto, es imposible que se hayan leído realmente esas tesis.

Para tratar de solventar todas estas cuestiones, se consultaron otras bases de datos como Dialnet <http:// dialnet.unirioja.es $>$, la Red de Bibliotecas Universitarias $<$ http://rebiun.absysnet.com>, el centro virtual Cervantes $<$ www.cervantesvirtual.com $>$ y el buscador Mastesis $<$ www.mastesis.com $>$. Asimismo, en los casos en los que no fue posible recuperar esa información, se acudió a las bases de datos de tesis doctorales de las bibliotecas de cada una de las universidades correspondientes.

Gracias a estas búsquedas se completó la información relativa al departamento de lectura. Sin embargo, ni la fecha exacta de lectura ni la composición del tribunal evaluador se pudieron recuperar en varios casos.

Una vez finalizado el proceso, la información de los 1020 registros seleccionados se introdujo en una base de datos creada ad hoc siguiendo las indicaciones de Fuentes y Arguimbau (2010), quienes se refieren a indicadores temáticos (áreas y disciplinas concretas de conocimiento) y de productividad (directores, centros de lectura, composición de los tribunales y años). Además, se tuvieron en cuenta otras variables que han recogido autores de estudios similares a éste (Martínez Pestaña, 2011a; 2011 b; Repiso, 2013) como el sexo del autor y el idioma en el que se ha escrito la tesis.
Asimismo, se clasificaron temáticamente las tesis analizadas utilizando para ello la división en cuatro grandes áreas de conocimiento del Ministerio de Educación, Cultura y Deporte del Gobierno de España. Estas áreas de conocimiento son: Ciencias Experimentales y de la Salud; Ciencias Sociales y Jurídicas; Humanidades; e Ingeniería y Tecnología

De este modo, la base de datos utilizada para analizar la información estaba compuesta por diez variables:

1) Nombre completo del autor;

2) Sexo del autor;

3) Título completo del trabajo;

4) Año de lectura;

5) Idioma de la tesis;

6) Área de conocimiento;

7) Universidad en la que se leyó la tesis;

8) Departamento al que estaba adscrito el doctorando;

9) Director o directores de la tesis;

10) Composición del tribunal.

\section{Resultados}

\section{Evolución de la producción global}

Las dos primeras tesis sobre Internet se defendieron en España en 1996. Se trata de la investigación de Luis García Pareras titulada "Internet y neurocirugía: diseño y prueba de una red neuroquirúrgica internacional", dirigida por Luis González Feria y defendida en el departamento de Cirugía de la Universidad de La Laguna; y de la de Jorge Bustinza Esparta titulada "Una propuesta metodológica para la integración de sistemas hipermedia en la enseñanza de la arquitectura. Su aplicación al estudio del hormigón armado", dirigida por Sebastián Dormido Bencomo y defendida en el departamento de Edificación de la Universidad de Navarra².

\footnotetext{
2 Determinar cuál de las dos fue la primera resulta una tarea imposible con los datos existentes en Teseo, ya que tanto en uno como en otro registro se producen las inexactitudes en el día y mes de lectura a las que se ha hecho referencia en el apartado anterior, es decir, aparece como fecha por defecto el 1 de enero del año correspondiente, a pesar de que la tesis realmente ha sido defendida en otro día distinto.
} 
Desde ese momento y hasta 2011 se han defendido en las universidades españolas 1020 tesis sobre Internet, lo que da una media de 63,75 tesis anuales. La tendencia general en la producción es al alza (Figura 1) y alcanza su máximo en el último año analizado, 2011, con 145 tesis doctorales defendidas. En los tres primeros años, la producción es exigua, y el primer despegue se produce en 1999. Otros repuntes importantes tienen lugar en los años 2002, 2003, 2009 y 2010. El crecimiento es constante año a año salvo dos excepciones: de 2004 a 2005 la producción pasa de 86 a 68 tesis doctorales; y entre 2006 y 2007 se produce un nuevo descenso, pasando de 79 a 69 tesis. A partir de ese año es cuando se produce el mayor aumento, de manera que entre 2008 y 2011 se han leído 486 tesis doctorales, casi la mitad de todo el periodo analizado (47,6\%). Además, 2011 es el año de mayor producción, con 145 tesis.

\section{Idioma y sexo de los doctorandos}

Las 1020 tesis sobre Internet defendidas en España hasta 2011 están escritas en ocho idiomas diferentes. El castellano es el idioma empleado de forma mayoritaria, ya que 842 tesis (82,5\% del total) están redactadas en esa lengua. El segundo idioma más presente es el inglés, que ha sido empleado en 132 tesis $(12,9 \%)$. El resto de lenguas empleadas tiene una presencia mucho menory son, por este orden, el catalán $(2,5 \%)$; el gallego $(0,9 \%)$, el portugués $(0,4 \%)$, el francés $(0,3 \%)$, el euskera $(0,2 \%)$ y el italiano $(0,1 \%)$.

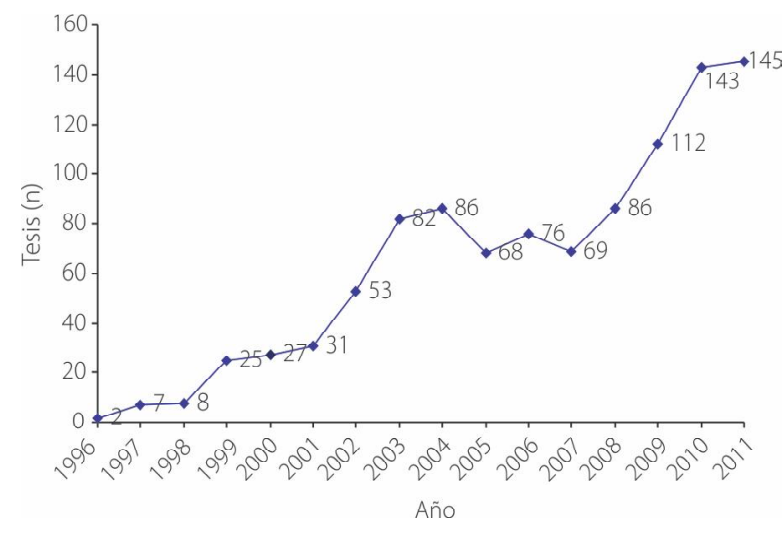

Figura 1. Evolución de la producción de tesis doctorales por años. Fuente: Elaboración propia (2015).
En cuanto al sexo de los doctorandos, los hombres representan el 61,3\% de las autorías, con 625 tesis doctorales, mientras que el 38,7\% restante (395 tesis) corresponde a mujeres. Examinando la distribución anual de autorías por sexo, los hombres lideran la producción en la mayoría de años analizados, salvo en 2004, en el que la cifra de autorías se distribuye al 50,0\% entre hombres y mujeres. A partir de ahí, las cifras de producción de tesis por uno y otro sexo suben y lo hacen de manera prácticamente uniforme (Figura 2).

\section{Áreas de conocimiento}

Algo más de la mitad de las tesis sobre Internet defendidas en España en el periodo analizado corresponden al área de Ciencias Sociales y Jurídicas, con 515 tesis $(50,5 \%$ del total). El segundo puesto corresponde al área de Ingeniería y Tecnología, con 343 tesis (33,6\%). A gran distancia se sitúan las áreas de Humanidades, con 102 tesis $(10,0 \%)$ y Ciencias Experimentales y de la Salud, con 60 tesis (5,9\%).

Existen varias diferencias significativas con la distribución de las cifras de producción total en el mismo periodo. En ese caso, el área de Ciencias Experimentales y de la Salud lidera la clasificación con casi la mitad de las tesis (49 721, un 46,3\%), seguida del área de Ciencias Sociales y Jurídicas, con 23.848 tesis (22,2\%); Humanidades, con 15298 tesis (14,2\%); e Ingeniería y Tecnología, con 13913 tesis (13,0\%). La producción total se completa con las 4581 tesis $(4,3 \%)$ no atribuibles a ninguna de las cuatro áreas de conocimiento.

\section{Universidades y departamentos de lectura}

\section{Universidades}

Las tesis localizadas se han defendido en 62 universidades, si bien en 32 de ellas se han leído 10 o menos tesis. La Universidad Complutense de Madrid es la que presenta una mayor producción con 123 tesis (12,06\% del total), seguida por la Politécnica de Catalunya con 68 (6,67\%), la Universidad Nacional de Educación a Distancia con 53 (5,20\%) y la Universidad Politécnica de Valencia con 50 (4,90\%). Hay otras dos universidades con más de 40 tesis leídas: son la Autónoma de Barcelona, con $47(4,61 \%)$ y la de Granada con 46 (4,51\%). 




Figura 2. Evolución de la producción de tesis doctorales por años según sexo.

Fuente: Elaboración propia (2015).

Como se puede ver, la mayoría de los primeros puestos están ocupados por las universidades más antiguas, si bien hay que resaltar la producción de algunos centros que comenzaron su andadura a finales de la década de los 80 o a principios de los 90 del siglo pasado. Destacan especialmente en este sentido las 28 tesis defendidas en la Carlos III (fundada en 1989), las 21 de Vigo (1990) o las 17 de la Oberta de Catalunya (1994).

Asimismo, a la luz de estos datos se deduce que de las 75 universidades públicas y privadas que forman parte de la Conferencia de Rectores de las Universidades Españolas (Crue), hay 13 en las que no se ha defendido ninguna tesis sobre Internet: Abat Oliba CEU, Alfonso X El Sabio, Antonio de Nebrija, Católica de Ávila, Católica de Valencia San Vicente Mártir, Universidad a Distancia de Madrid, Universidad Europea Miguel de Cervantes, Francisco de Vitoria, Universidad Internacional de Andalucía, Universidad Internacional Menéndez Pelayo, Universidad Internacional de La Rioja, San Jorge y Vic.
En algunos casos, este hecho se debe a su relativamente reciente fundación, los estudios de tercer ciclo no se han implantado o se acaban de implantar. De hecho, ninguna de ellas cuenta todavía con ninguna tesis registrada en Teseo. Es el caso de la Universidad Europea Miguel de Cervantes, Universidad a Distancia de Madrid y Universidad Internacional de La Rioja.

Otras universidades que sí cuentan ya con estudios de doctorado y con tesis defendidas, como se puede corroborar en Teseo, pero en las que no se ha defendido ninguna sobre Internet, son Abat Oliba CEU, Alfonso X El Sabio, Antonio de Nebrija, Católica de Ávila, Católica de Valencia San Vicente Mártir, Francisco de Vitoria, San Jorge y Vic. No obstante, en varias de ellas el número total de tesis defendidas ni siquiera supera la decena. Por último, en el caso de dos de ellas, la Universidad Internacional de Andalucía y la Universidad Internacional Menéndez Pelayo, es su propia estructura y las características de su oferta formativa, en la que no se incluye la defensa de tesis doctorales, la que explica este hecho. 


\section{Departamentos}

Para complementar y precisar la información anterior, se analizan a continuación cuáles han sido los departamentos con un mayor de tesis dirigidas, ya que es un dato que aparece en los registros de Teseo, a diferencia de lo que ocurre con la facultad, una información que no se encuentra en esa base de datos. Destacan especialmente por su producción el Departamento de Didáctica, Organización Escolar y Didácticas Especiales de la Universidad Nacional de Educación a Distancia (Uned), con 22 tesis; el Departamento de Periodismo II (Estructura y Tecnología de la Información) de la Complutense con 20 tesis; el Departamento de Arquitectura de Computadores de la Politécnica de Catalunya; y el Internet Interdisciplinary Institute de la Oberta de Catalunya, ambos con 17 tesis.

La mayoría de ellos pertenecen a las universidades que se situaban en los primeros lugares en el apartado anterior, con excepciones como el mencionado departamento de la Oberta de Catalunya, cuya propia naturaleza se centra precisamente en el estudio de Internet. También destaca la presencia de hasta tres departamentos de la Politécnica de Catalunya en los seis primeros puestos de la tabla; y la producción del Departamento de Ingeniería Telemática de la Universidad de Vigo, que con diez tesis engloba casi la mitad de la producción de su universidad (21 tesis). Asimismo, se puede resaltar la presencia de hasta tres departamentos de la Facultad de Ciencias de la Información de la Universidad Complutense, Periodismo II, Comunicación Audiovisual y Publicidad I y Periodismo IV.

\section{Dirección de tesis}

Un total de 1078 directores han dirigido las 1020 tesis analizadas. Uno de los motivos que explica el hecho de que el número de directores sea más alto que el de tesis doctorales reside en la cifra de codirecciones, ya que 280 de las tesis analizadas han sido codirigidas. Asimismo, 13 de las tesis han contado con tres directores. Por tanto, 293 tesis, un 28,7\% del total, han tenido dos o más directores. Mientras, las 727 restantes $(71,3 \%)$ contaron con un único director.

El año que registra un mayor número de tesis con más de un director es 2011, en el que 56 tesis fueron codirigidas y otras seis tuvieron tres directores. Ese año es el que presenta también un mayor porcentaje de tesis con más de un director, por cuanto esas 62 tesis representan el 42,7\% de las 145 tesis presentadas en el periodo. Asimismo, sólo 161 profesores (14,9\% del total) han dirigido dos o más tesis, mientras que los 917 (85,1\%) restantes han dirigido una única tesis.

En la Tabla 2 aparecen los profesores que han dirigido cuatro o más tesis. A la cabeza de todos ellos, con 13 tesis dirigidas, el profesor Domingo José Gallego Gil, del Departamento de Didáctica, Organización Escolar y Didácticas Especiales de la Universidad Nacional de Educación a Distancia, que es precisamente el departamento con mayor producción de todos los analizados

En segundo lugar se sitúa José Vicente Rodríguez Muñozdel Departamento de Informacióny Documentación de la Universidad de Murcia, y que ha sido decano de su facultad durante varios años.

Como puede verse, la mayoría de ellos pertenecen a las universidades y departamentos que aparecen en los primeros puestos de la tabla del apartado anterior, con la única excepción del profesor José María Malgosa Sanahuja, del Departamento de Tecnologías Industriales de la Universidad Politécnica de Cartagena, con cuatro tesis dirigidas. Por otro lado, de los 17 profesores que aparecen en la tabla, 12 son Catedráticos y los otros 5 son Profesores Titulares.

Los datos relativos a las direcciones de las tesis examinadas se completan con otros 37 profesores que han dirigido tres tesis. Igualmente, un total de 107 profesores han dirigido dos tesis.

\section{Presencia en tribunales y presidencias}

Lo primero que hay que aclarar en este apartado es que no se ha podido recuperar la información completa relativa la composición de los tribunales de las tesis analizadas, ya que en Teseo faltaban los nombres del presidente en una de ellas y del secretario en diez tesis. Asimismo, en 57 de las tesis no figuran los nombres de los vocales, y en otra tesis no aparece el nombre de ninguno de los tres vocales. En total, han quedado sin identificar 128 identidades de miembros de los tribunales. 
Tabla 2. Directores con cuatro o más tesis dirigidas.

\begin{tabular}{lcll}
\hline Director & Tesis (n) & Universidad & Departamento \\
\hline Gallego Gil, Domingo José & 13 & Uned & Didáctica, Organización Escolar y Didácticas Especiales \\
Rodríguez Muñoz, José Vicente & 7 & Murcia & Información y Documentación \\
Fernández del Moral, Javier & 6 & Complutense & Estructura y Tecnología Información \\
Ruipérez García, Germán & 6 & Uned & Filologías Extranjeras y sus Lingüísticas \\
García García, Francisco & 5 & Complutense & Comunicación Audiovisual y Publicidad II \\
Paradells Aspas, Josep & 5 & Politécnica Catalunya & Ingeniería Telemática \\
Adelantado Mateu, Eulalia & 4 & Politécnica Valencia & Comunicación Audiovisual, Documentación e Historia del Arte \\
Castells Oliván, Manuel & 4 & Oberta Catalunya & Internet Interdisciplinary Institute \\
Diaz Nosty, Bernardo & 4 & Málaga & Periodismo \\
Dormido Bencomo, Sebastián & 4 & Uned & Informática y Automática \\
Flavián Blanco, Carlos & 4 & Zaragoza & Economía y Dirección de Empresas \\
Lallana García, Fernando & 4 & Complutense & Estructura y Tecnología Información \\
López Muñoz, Francisco Javier & 4 & Málaga & Lenguaje y Ciencias de la Computación \\
Malgosa Sanahuja, Jose María & 4 & Politécnica Cartagena & Tecnologías Industriales \\
Moya Anegón, Felix & 4 & Granada & Biblioteconomía y Documentación \\
Pelechano Ferrragud, Vicente & 4 & Politécnica Valencia & Sistemas Informáticos y Computación \\
Ródenes Adam, Manuel & 4 & Politécnica Valencia & Organización de Empresas \\
\hline
\end{tabular}

Fuente: Elaboración propia (2015).

Nota: Uned: Universidad Nacional de Educación a Distancia.

Tabla 3. Académicos con al menos ocho presencias o seis presidencias en tribunales.

\begin{tabular}{|c|c|c|c|}
\hline Nombre & Universidad & Presencias & Presidencias \\
\hline Moreiro González, José Antonio & Carlos III & 21 & 14 \\
\hline Esteve Ramírez, Francisco & Complutense de Madrid & 12 & 4 \\
\hline Caridad Sebastián, Mercedes & Carlos III & 11 & 8 \\
\hline Fernández Del Moral, Javier & Complutense de Madrid & 11 & 10 \\
\hline Soriano Ibáñez, Miguel & Politécnica Catalunya & 11 & 2 \\
\hline García Haro, Juan & Politécnica Cartagena & 10 & 5 \\
\hline Bigné Alcañiz, Enrique & Valencia & 9 & 1 \\
\hline Vázquez Casielles, Rodolfo & Oviedo & 9 & 3 \\
\hline Azcorra Saloña, Arturo & Politécnica de Madrid & 8 & 6 \\
\hline Bartolome Crespo, Donaciano & Complutense de Madrid & 8 & 0 \\
\hline Cabero Almenara, Julio & Sevilla & 8 & 6 \\
\hline Martin Armario, Enrique & Sevilla & 8 & 3 \\
\hline Medina Rivilla, Antonio & Uned & 8 & 7 \\
\hline Sallent Ribes, Sebastià & Politécnica Catalunya & 8 & 4 \\
\hline Cebrián Herreros, Mariano & Complutense de Madrid & 6 & 6 \\
\hline Domínguez Garrido, María Concepción & Uned & 6 & 6 \\
\hline Mairal Usón, Ricardo & Uned & 6 & 6 \\
\hline Pazos Sierra, Juan & A Coruña & 6 & 6 \\
\hline
\end{tabular}

Fuente: Elaboración propia (2015).

Fonte: Uned: Universidad Nacional de Educación a Distancia. 
El número de académicos presentes en al menos en algún tribunal asciende a 3614 . En la Tabla 3 se muestra a los profesores que han estado presentes en al menos ocho tribunales o que han presidido al menos seis de ellos.

En el primer y en el tercer lugar se sitúan dos profesores de la Universidad Carlos III, José Antonio Moreiro González y Mercedes Caridad Sebastián, con 21 y 11 presencias respectivamente. El dato habla del prestigio individual de ambos, teniendo en cuenta la menor producción de su universidad (28 tesis) en comparación con otras a las que pertenecen otros profesores que aparecen en la tabla. Así, el resto de los que ocupan los primeros lugares sí que pertenecen a universidades con una mayor producción de tesis. Es el caso de Francisco Esteve Ramírez (12 presencias) y Javier Fernández del Moral (11); ambos de la Universidad Complutense de Madrid; y de Miguel Soriano Ibáñez (11 presencias) de la Universidad Politécnica de Catalunya.

Además, en el caso de algunos de ellos, la mayoría de estas presencias en tribunales han tenido lugar en tesis que no se defendían en sus respectivas universidades de origen. Así sucede en el caso del profesor Moreiro González, 17 de cuyas 21 presencias se producido fuera de la Universidad Carlos III; o de la profesora Caridad Sebastián, con siete de sus 11 presencias fuera de su centro de origen (también la Universidad Carlos III); o del profesor Soriano Ibáñez, con sólo una presencia en tribunales de tesis leídas en la Universidad Politécnica de Catalunya.

Otros casos que destacan, a tenor de los datos de producción de sus respectivas universidades, son los de Juan García Haro, de la Politécnica de Cartagena, con 10 presencias; Rodolfo Vázquez Casielles, de la Universidad de Oviedo, con nueve presencias; y el de Juan Pazos Sierra, de la Universidad de La Coruña, con seis. Por otro lado, de los 18 profesores que aparecen en la tabla, 17 son Catedráticos y uno es Titular de Universidad.

\section{Discusión}

Los datos anteriores permiten deducir que Internet, como objeto de estudio, se encuentra en pleno proceso de consolidación y que, a pesar de contar todavía - como es lógico - con poca tradición, existe un creciente interés por su estudio en la universidad española. Las 1020 tesis defendidas en el periodo analizado así lo corroboran, pero también los datos relativos a la producción por años, y en especial el hecho de que entre 2008 y 2011 se hayan leído 486 tesis, es decir casi la mitad de todas las analizadas $(47,6 \%)$.

Este ritmo de crecimiento en la producción de tesis sobre Internet es sensiblemente superior al experimentado en la producción general de tesis en el periodo analizado. Así, por ejemplo, entre los cursos académicos 2007-2008 y 2010-2011 se defendieron en España 32728 tesis, es decir el 32,33\% del total, un porcentaje inferior al $47,60 \%$ de tesis sobre Internet leídas más o menos en el mismo periodo ${ }^{3}$.

La distribución de autorías por sexos muestra un mayor número de hombres, con un 61,3\% del total, un porcentaje superior al que muestra la producción anual en el periodo analizado (curso 1996/97 al curso 2010/11). Según los datos del INE, hay allí un equilibrio mucho mayor, ya que del total de 107361 tesis doctorales defendidas en las universidades españolas, 57561 (un 53,7\%) corresponden a autores y 49710 (un 46,3\%) a mujeres. Además, la evolución por años muestra una tendencia a que esos datos se igualen, con un porcentaje de mujeres en ascenso prácticamente constante, hasta situarse en el 49,1\% en el último de los cursos académicos estudiados, 2010-2011.

Sin embargo, esa tendencia no se da en el caso de las tesis sobre Internet. Estehecho guarda probablemente una estrecha relación con la distribución de esas tesis por áreas de conocimiento y la preponderancia de las Ciencias Sociales, que es precisamente el área en el que las mujeres tienen una menor presencia porcentual en la producción total de tesis: de las 23848 tesis doctorales defendidas en ese campo de conocimiento durante el periodo analizado, sólo algo más de una tercera parte, 8558 (es decir, un 35,9\%), corresponden a mujeres.

Estas cifras coinciden con la tendencia mundial que muestran Larivière et al. (2013), quienes realizaron un análisis bibliométrico de todos los artículos que

\footnotetext{
${ }_{3}$ Mientras los datos estadísticos extraídos del INE aparecen clasificados por cursos académicos, las tesis doctorales están distribuidas en Teseo por años naturales, de ahí que no pueda comparar exactamente el mismo periodo. Sin embargo, la comparación de los dos porcentajes resulta bastante ilustrativa.
} 
aparecen en tres de las bases de datos de Web of Science "Science Citation Index Expanded, Social Science Citation Index y Arts and Humanities Citation Index" entre 2008 y 2012. Estos autores concluyeron que sigue existiendo un desequilibrio, y que la desigualdad de género continúa siendo la tónica general en la investigación científica.

En otro orden de cosas, la mayoría de las tesis analizadas (82,5\%) están escritas en castellano, mientras que destaca el porcentaje de trabajos escritos en inglés (12,9\%). Aunque este dato no aparece en la información del INE, y por tanto no se puede comparar con la producción total, sí que es un porcentaje mayor que en otros estudios similares que han estudiado este apartado (Martínez Pestaña, 2011a;2011b; Repiso, 2013). En cuanto a las seis lenguas restantes, su uso es minoritario.

Las Ciencias Sociales y Jurídicas son el campo en el que más ha proliferado la investigación sobre Internet en España, ya que más de la mitad de tesis analizadas corresponden a esa área, por encima de las tesis correspondientes a Ingeniería y Tecnología, que son aproximadamente una tercera parte del total. Las tesis de las áreas de Humanidades y de las Ciencias Experimentales y de la Salud tienen una presencia mucho menor. El dato contrasta con el de la producción total de tesis en España durante los años analizados, en la que es precisamente el campo de las Ciencias Experimentales y de la Salud el que muestra una mayor producción.

Particularmente destacable resulta el hecho de que haya más tesis correspondientes a Ciencias Sociales y Jurídicas que a Ingeniería y Tecnología, que apunta a un mayor interés por el estudio de Internet desde esa perspectiva. Una tendencia que iría en la línea señalada por uno de los mayores estudiosos de la Red, el sociólogo Castells (2001, p.1), quien pone de manifiesto que Internet "es mucho más que una tecnología. Es un medio de comunicación, de interacción y de organización social".

Asimismo, estos datos muestran que Internet, a pesar de constituir una línea de investigación nueva, goza ya de una presencia predominante en algunas áreas, especialmente aquellas en las que la información es uno de sus objetos de estudio. Del mismo modo, los datos obtenidos confirman la importancia de que Internet esté presente de manera transversal en los plantes de formación de las diferentes disciplinas, y consolidan el concepto de alfabetización informacional como medio para el aprendizaje permanente y la generación de conocimientos (Uribe Tirado, 2010a; 2010b), por lo que parece lógico pensar que debería ser considerada como una de las competencias clave para cualquier persona a la hora de desarrollar las políticas científicas y educativas en España.

Por otro lado, el número y porcentaje de tesis defendidas en cada universidad también muestra algunas diferencias respecto a los datos totales que reflejaba, por ejemplo, el trabajo de Fuentes y Arguimbau (2010). En ambos casos, es la Universidad Complutense de Madrid la que lidera de manera destacada la producción, pero en el resto de lugares destacados de una y otra tabla hay diferencias significativas. De hecho, las universidades que ocupan los lugares segundo al cuarto en la producción de tesis sobre Internet, como la Politécnica de Catalunya, la Uned o la Politécnica de Valencia, no aparecen siguiera entre los diez primeros puestos de la producción general de tesis en España (Fuentes \&Arguimbau, 2010). Por el contrario, universidades como la de Barcelona, Valencia o la Autónoma de Madrid, que ocupaban un lugar bastante destacado en esa tabla de producción general, cuentan con un número de tesis más limitado en lo referente a Internet.

Los datos relativos a la dirección de las tesis muestran, en primer lugar, la misma diversidad que la distribución de tesis entre universidades, ya que son 1078 los profesores que han dirigido al menos alguna tesis de las analizadas. Del mismo modo, los directores con mayor producción son profesores e investigadores de gran prestigio en sus respectivas áreas de conocimiento, ya que la mayoría de ellos son catedráticos y el resto profesores titulares.

Asimismo, la codirección es un fenómeno creciente y que aumenta de forma progresiva. En efecto, casi el 30,0\% igualar as casas de las tesis analizadas hayan contado con dos o más directores y que en 2011, el último año que abarca esta investigación, ese porcentaje ascienda al 42,7\%.

Mientras, el prestigio es también la nota común de los profesores que han estado presentes en un mayor 
número de tribunales, ya que la mayoría de ellos son catedráticos. Además, este hecho viene corroborado también por el caso de que varios de ellos hayan sido reclamados para formar parte de tribunales de tesis que en la mayoría de las ocasiones se han leído en otras universidades distintas a las propias.

El prestigio es, por tanto, una de las características de la Universidad Complutense que ocupa el primer lugar en el ranking por universidades, de los directores con mayor producción y de los profesores que han formado parte de más tribunales. En consecuencia, la sociología de la ciencia nos aporta algunos de los factores que explican estos resultados, como por ejemplo el llamado efecto Mateo (Merton, 1968), que puede definirse como una regla no escrita que funciona en la comunidad científica y según la cual, precisamente, el prestigio llama de forma automática a más prestigio, es decir, la influencia de una investigación viene determinada, al menos en parte, por el prestigio previo de los autores y las instituciones que las llevan a cabo.

Algo parecido cabe decir del concepto de capital acumulado (Bourdieu, 2003), según el cual el prestigio de las instituciones en las que se han formado o el del director de su tesis, entre otros, son elementos que ayudan al científico a obtener el reconocimiento de sus pares. En consecuencia, los estudiantes de doctorado españoles parecen tener muy en cuenta este prestigio y estos conceptos a la hora de elegir el tema, el director y el lugar para desarrollar su tesis, y se trata de factores que resultan decisivos a pesar de hablar de un objeto de estudio relativamente novedoso como Internet.

\section{Referencias}

Bourdieu, P. Los usos sociales de la ciencia. Buenos Aires: Nueva Visión, 2003.

Castells, M. Internet y la sociedad red. In: Conferencia de Presentación del Programa de Doctorado sobre la Sociedad de la Información y el Conocimiento. Barcelona: Universidad Oberta de Catalunya, 2001. Disponible en: <https://engage. intel.com/servlet/JiveServlet/ download Body/26111-102-131790/INTERNET\%20Y\%20LA\%20SOCIEDAD\% 20RED.pdf>. Acceso en: 10 mar. 2014.

Delgado, E. et al. Análisis bibliométrico y de redes sociales aplicado a las tesis bibliométricas defendidas en España (1976-2002): temas, escuelas científicas y redes académicas. Revista Española de Documentación Científica, v.29, n.4 p.493-524, 2006. <http://dx.doi.org/10.3989/redc.2006.v29.i4>.

\section{Conclusión}

La investigación sobre Internet en las universidades españolas esta recibiendo una atención creciente, y la tendencia para los próximos años parece apuntar en esa línea. El número de tesis defendidas es alto y el porcentaje de aumento año a año supera al que registra la producción total.

La comparativa entre la producción de tesis sobre Internet y la producción total en lo que respecta a los diversos indicadores analizados muestra algunas semejanzas (por ejemplo, en cuanto a la diversificación de la producción entre un número muy amplio de centros o en el perfil de los directores y miembros de los tribunales), aunque también algunas diferencias significativas (sobre todo en el área de conocimiento y en las universidades que registran una mayor producción).

Por último, teniendo en cuenta precisamente esa tendencia creciente y que solo hablamos de un periodo de 15 años, sería interesante repetir este estudio en el futuro para comprobar si estas conclusiones obtenidas de la presente investigación se consolidan o, por el contrario, varían con el paso del tiempo.

Agradecimientos

Esta investigación ha sido parcialmente financiada por Universidad Internacional de la Rioja, dentro del Plan Propio de Investigación, Desarrollo e Innovación (2013-2015).
Díaz-Campo, J. La investigación sobre Internet en las facultades de Comunicación españolas. Análisis bibliométrico de tesis doctorales (1997-2012). Documentación de las Ciencias de la Información, v.37, p.305-320, 2014. <http://dx.doi.org/ 10.5209/rev_DCIN.2014.v37.46828>

Fuentes, E.; Arguimbau, L. Las tesis doctorales en España (1997/2008): análisis, estadísticas y repositorios cooperativos. Revista Española de Documentación Científica, v.33, n.1, p.63-89, 2010. <http://dx.doi.org/10.3989/redc.2010.1.711>.

Larivière, V. et al. Bibliometrics: Global gender disparities in science. Nature, v.504, n.7479, p.211-213, 2013.

Marcos Recio, J.C.; Martínez Pestaña, M.J.; Blasco López, M.F. Producción y dirección de tesis doctorales sobre publicidad en la universidad española (1971-2010). Revista Española de 
Documentación Científica, v.35, n.3, p.433-452, 2012. <http:// dx.doi.org/10.3989/redc.2012.3.890>.

Martínez Pestaña, M.J. La producción de tesis doctorales sobre temas publicitarios (1971-2001). Documentación de las Ciencias de la Información, v.27, p.237-267, 2004.

Martínez Pestaña, M.J. Evaluación de la producción científica sobre publicidad (1971-2001). 2011. Tesis (Doctoral em Documentación: Fundamentos, Tecnologias y Aplicaciones) Facultad de Ciencias de la Información, Universidad Complutense de Madrid, Madrid, 2011a.

Martínez Pestaña, M.J. La investigación universitaria en Publicidad: producción y temática de las tesis doctorales (1971-2001). Documentación de las Ciencias de la Información, v.34, p.119-156, 2011 b.

Merton, R.K. The Matthew effect in science. Science, v.159, n.3810, p.56-63, 1968.

Moralejo Álvarez, M.R. Las tesis doctorales en las universidades españolas, Control bibliográfico y acceso. Revista General de Información y Documentación, v.10, n.1, p.235-243, 2000.

Oliva Marañón, C. Visibilidad y producción científica de las Tesis Doctorales de Historia del Periodismo en las Universidades Españolas (2002-2012). Historia y Comunicación Social, v.19, p.183-193, 2014. <http://dx.doi.org/10.5209/ rev_HICS.2014.v19.45020>.

Orera Orera, L. Bibliotecas digitales de tesis doctorales: metodología para su planificación. Boletín de la Asociación Andaluza de Bibliotecarios, v.72, p.55-72, 2003.

Repiso, R. La investigación en televisión, cine, radio y fotografía en España: análisis bibliométrico y de redes sociales de la estructura científica. 2013. Tesis (Doctoral) - Departamento de Información y Comunicación, Universidad de Granada, 2013.
Repiso, R.; Delgado, E.; Torres, D. Análisis bibliométrico de la producción española de Tesis Doctorales sobre Cine 1978-2007. In: Bort Gual, I.; García Catalán, S.; Martín Núñez, M. (Ed.). Actas del IV Congreso Internacional sobre Análisis Fílmico: Nuevas Tendencias e Hibridaciones de los Discursos Audiovisuales en la Cultura Digital Contemporánea. Madrid: Ediciones de las Ciencias Sociales, 2012a. p.976-987.

Repiso, R.; Torres, D.; Delgado, E. Análisis bibliométrico y de redes sociales en tesis doctorales españolas sobre televisión (1976/2007). Comunicar, v.37, p.151-159, 2011.

Repiso, R.; Torres, D.; Delgado, E. Análisis de la investigación sobre radio en España: una aproximación a través del análisis bibliométrico y de redes sociales de las tesis doctorales defendidas en España entre 1976-2008. Estudios sobre el Mensaje Periodístico, v.17, n.2, p.417-429, 2012b.

Sánchez-Vigil, J.M.; Marcos-Recio, J.C.; Olivera-Zaldua, M. Tesis doctorales sobre fotografía en la universidad española. Análisis de la producción y dirección (1976-2012). Revista Española de Documentación Científica, v.37, n.1, e034, p.1-14, 2014. <http:// dx.doi.org/10.3989/redc.2014.1.1073>.

Sorli, Á.; Merlo, J.A. Bases de datos y recursos en Internet de tesis doctorales. Revista Española de Documentación Científica, v.25, n.1, p.95-106, 2002. <http://dx.doi.org/10.3989/redc. 2002.v25.i1>.

Uribe Tirado, A. La alfabetización informacional en la universidad. Descripción y categorización según los niveles de integración de ALFIN. Caso Universidad de Antioquia. Revista Interamericana de Bibliotecología, v.33, n.1, p.10-45, 2010a.

Uribe Tirado, A. La alfabetización informacional en Iberoamérica. Ibersid, v.4, p.165-176, 2010b. 